\title{
OPERATORS ASSOCIATED WITH A PAIR OF NONNEGATIVE MATRICES
}

GERALD E. SUCHAN

\begin{abstract}
Let $A_{m \times n}, B_{m \times n}, X_{n \times 1}$, and $Y_{m \times 1}$ be matrices whose entries are nonnegative real numbers and suppose that no row of $A$ and no column of $B$ consists entirely of zeroes. Define the operators $U, T$ and $T^{\prime}$ by $(U X)_{i}=X_{i}^{-1}$ [or $(U Y)_{i}=Y_{i}^{-1}$ ], $T=U B^{t} U A$ and $T^{\prime}=U A U B^{t} . T$ is called irreducible if for no nonempty proper subset $S$ of $\{1, \cdots, n\}$ it is true that $X_{i}=0, i \in S ; X_{i} \neq 0, i \notin S$, implies $(T X)_{i}=0, i \in S ;(T X)_{i} \neq 0, i \notin S$. M. V. Menon proved the following Theorem. If $T$ is irreducible, there exist row-stochastic matrices $A_{1}$ and $A_{2}$, a positive number $\theta$, and two diagonal matrices $D$ and $E$ with positive main diagonal entries such that $D A E=A_{1}$ and $\theta D B E=A_{2}^{t}$. Since an analogous theorem holds for $T^{\prime}$, it is natural to ask if it is possible that $T^{\prime}$ be irreducible if $T$ is not. It is the intent of this paper to show that $T^{\prime}$ is irreducible if and only if $T$ is irreducible.
\end{abstract}

Suppose that each of $m$ and $n$ is a positive integer. Let $A_{m \times n}$ and $B_{m \times n}$ be matrices whose entries are nonnegative real numbers and suppose that no row of $A$ and no column of $B$ consists entirely of zeroes. Let $X_{n \times 1}$ and $Y_{m \times 1}$ be matrices whose entries are taken from the extended real nonnegative numbers. Define the operator $U$ by $(U X)_{i}=X_{i}^{-1}$ [or $(U Y)_{i}=$ $\left.Y_{i}^{-1}\right]$ and let $0^{-1}=\infty, \infty^{-1}=0, \infty+\infty=\infty, 0 \cdot \infty=0$, and if $\alpha>0, \alpha \cdot \infty=$ $\infty$ [1]. Define the operators $T$ and $T^{\prime}$ by $T=U B^{t} U A$ and $T^{\prime}=U A U B^{t}$ where $B^{t}$ is the transpose of $B$. Clearly

$$
(T X)_{i}=\left(\sum_{j=1}^{m} b_{j i}\left(\sum_{k=1}^{n} a_{j k} X_{k}\right)^{-1}\right)^{-1} .
$$

$T$ is called irreducible if for no nonempty proper subset $S$ of $N=\{1, \cdots, n\}$ is it true that $X_{i}=0, i \in S ; X_{i} \neq 0, i \notin S$, implies $(T X)_{i}=0, i \in S ;(T X)_{i} \neq 0$, $i \notin S . T^{\prime}$ is defined to be irreducible analogously.

$\mathrm{M}$. V. Menon [2] proved the following.

THEOREM 1. If $T$ is irreducible, then there exist row-stochastic matrices $A_{1}$ and $A_{2}, a$ positive number $\theta$, and two diagonal matrices $D$ and $E$ with positive main diagonal entries such that $D A E=A_{1}$ and $\theta D B E=A_{2}^{t}$.

Received by the editors March 5, 1971 .

AMS 1970 subject classifications. Primary 15A48; Secondary 15A15, 15A51.

Key words and phrases. Nonnegative matrix, stochastic, irreducible.

i American Mathematical Society 1972 
Since an analogous theorem holds for $T^{\prime}$, one would hope that $T^{\prime}$ might be irreducible even if $T$ is not. However, it is the intent of this paper to prove the following result.

THEOREM 2. $T^{\prime}$ is irreducible if and only if $T$ is irreducible.

Proof. If $T^{\prime}$ is not irreducible then there is a nonempty proper subset $S$ of $M=\{1, \cdots, m\}$ which has the property that if $Y_{m{ }^{\prime 1}}$ is such that $Y_{i}=0, i \in S, Y_{i} \neq 0, i \notin S$, then $\left(T^{\prime} Y\right)_{i}=0, i \in S,\left(T^{\prime} Y\right)_{i} \neq 0, i \notin S$. Let $Z_{n \times 1}=U B^{t} Y$, put $E=\left\{i \in N: Z_{i}=\infty\right\}$, and let $E^{\prime}$ be the complement of $E$ in $N$.

(1) If $i_{0} \in S$ then there exists $j_{0} \in N$ such that $a_{i_{0} j_{0}}\left(\sum_{k=1}^{m} b_{k j_{0}} Y_{k}\right)^{-1}=\infty$. Hence $Z_{j_{0}}=\infty$ and thus $E$ is not null.

(2) Let $S^{\prime}$ be the complement of $S$ in $M$ so that if $i_{0} \in S^{\prime}$, then there exists $j_{0} \in N$ such that $\infty>a_{\iota_{0} j_{0}}\left(\sum_{k=1}^{m} b_{k j_{0}} Y_{k}\right)^{-1}>0$. Hence $\infty>Z_{j_{0}}>0$ and $E^{\prime}$ is not null.

(3) Let $X_{n \times 1}$ be defined by putting $\infty>X_{i}>0$ if $i \in E$ and $X_{i}=0$ if $i \in E^{\prime}$. If $X_{i_{0}}=0$ then $\infty>\left(\sum_{j=1}^{m} b_{j i_{0}}\left(\sum_{k=1}^{m} a_{j k} Z_{k}\right)^{-1}\right)^{-1}>0$ and hence there exists $j_{0} \in M$ such that $\infty>b_{j_{0} i_{0}}>0$. Thus $\infty>\sum_{k=1}^{n} a_{j_{0} k} Z_{k}=\sum_{k \in E^{\prime}} a_{j_{0} k} Z_{k}+$ $\sum_{k \in E^{\prime}} a_{j_{0}} k Z_{k}>0$ so that $a_{j_{0} k}=0$ for $k \in E$. Therefore, if $X_{i_{0}}=0$, then $(T X)_{i_{0}}=0$.

(4) For $i_{0} \in E$, put $F=\left\{j \in M: b_{j i_{0}}=0\right\}$ and let $F^{\prime}$ be the complement of $F$ in $M$. Since $\infty>X_{i_{0}}>0$ then $(T Z)_{i_{0}}=\infty$ so that

$$
\sum_{j=1}^{m} b_{j i_{0}}\left(\sum_{k=1}^{n} a_{j k} Z_{k}\right)^{-1}=\sum_{j \in \boldsymbol{F}^{\prime}} b_{j i_{0}}\left(\sum_{k=1}^{n} a_{j k} Z_{k}\right)^{-1}+\sum_{j \in F^{\prime}} b_{j i_{0}}\left(\sum_{k=1}^{n} a_{j k} Z_{k}\right)^{-1}=0,
$$

and hence there exists $j_{0} \in F^{\prime}$ so that $b_{j_{0} i_{0}} \neq 0$. Thus $\sum_{k=1}^{n} a_{j_{0} i_{i}} Z_{k}=\infty$ so that there exists $k_{0} \in E$ such that $\infty>a_{j_{0} k_{0}} X_{k_{0}}>0$. Therefore if $\infty>X_{i_{0}}>0$ then $\infty>(T X)_{i_{0}}>0$.

It immediately follows from (1), (2), (3), and (4) that $T^{\prime}$ is irreducible if $T$ is irreducible. A similar argument proves that $T$ is irreducible if $T^{\prime}$ is irreducible.

\section{REFERENCES}

1. R. A. Brualdi, S. V. Parter and H. Schneider, The diagonal equivalence of a nonnegative matrix to a stochastic matrix, J. Math. Anal. Appl. 16 (1966), 31 50. MR 34 \#5844.

2. M. V. Menon, Sorme spectral properties of an operator associated with a pair of nonnegative matrices, Trans. Amer. Math. Soc. 132 (1968), 369-375. MR 37 \#1394.

Department of Ma rhfmatics, University of Houston, Houston, Texas 77004 